\title{
Anatomy of the Higgs Boson Decay into Two Photons in the Unitary Gauge
}

\author{
Athanasios Dedes and Kristaq Suxho \\ Division of Theoretical Physics, University of Ioannina, 45110 Ioannina, Greece \\ Correspondence should be addressed to Athanasios Dedes; adedes@cc.uoi.gr
}

Received 10 December 2012; Revised 4 April 2013; Accepted 17 May 2013

Academic Editor: Alexey Petrov

Copyright ( 2013 A. Dedes and K. Suxho. This is an open access article distributed under the Creative Commons Attribution License, which permits unrestricted use, distribution, and reproduction in any medium, provided the original work is properly cited.

\begin{abstract}
We review and clarify computational issues about the $W$-gauge boson one-loop contribution to the $H \rightarrow \gamma \gamma$ decay amplitude, in the unitary gauge and in the Standard Model. We find that highly divergent integrals depend upon the choice of shifting momenta with arbitrary vectors. One particular combination of these arbitrary vectors reduces the superficial divergency down to a logarithmic one. The remaining ambiguity is then fixed by exploiting gauge invariance and the Goldstone Boson Equivalence Theorem. Our method is strictly realised in four dimensions. The result for the amplitude agrees with the "famous" one obtained using dimensional regularisation (DR) in the limit $d \rightarrow 4$, where $d$ is the number of spatial dimensions in Euclidean space. At the exact equality $d=4$, a three-sphere surface term appears that renders the Ward Identities and the equivalence theorem inconsistent. We also examined a recently proposed four-dimensional regularisation scheme and found agreement with the DR outcome.
\end{abstract}

\section{Introduction}

Today one of the main focal points at the Large Hadron Collider (LHC) is to search for the Higgs boson $(H)$ [1-3] through its decay into two photons, $H \rightarrow \gamma \gamma$ (for reviews see $[4,5])$. Indeed, the recent $[6,7]$ observation by ATLAS and CMS experiments of a resonance, which could be the Standard Model (SM) Higgs boson, is based on data mainly driven by $H \rightarrow \gamma \gamma$. In the SM [8-10], this particular decay process goes through loop-induced diagrams involving either charged fermions or $W$-gauge bosons. Their calculation was first performed in [11] in the limit of light Higgs mass $m_{H} \ll m_{W}$, using dimensional regularisation in the 't HooftFeynman gauge. Since then, there are numerous works spent on improving this calculation including finite Higgs mass effects in linear and nonlinear gauges [12-14], different regularisation schemes [15-18], and/or different gauge choices [19].

The $H \rightarrow \gamma \gamma$ amplitude is originated, in broken (unbroken) phase, by a dimension-5 (dimension-6) SM gauge invariant operator(s) and, therefore, its expression, within a renormalizable theory, must be finite, gauge invariant and independent of any gauge choice. The amplitude should also be consistent with the Goldstone Boson Equivalence Theorem (GBET) [20-22] since the SM is a renormalizable, spontaneously broken, gauge field theory.

A problem arises when the $W$-gauge boson contribution (see Figure 1) to $H \rightarrow \gamma \gamma$ produces "infinite" results at intermediate steps. These problems are usually treated by using a gauge invariant regulator method, for example, dimensional regularization. In the unitary gauge [23], this indeterminacy is more pronounced and more difficult to handle with (however, using DR and unitary gauge with modern computer algorithms, this may not be a hard problem today [19]) due to the particular form of the $W$-gauge boson propagator. On the other hand it is much simpler to work with only few diagrams that involve physical particle masses, rather than many.

More specifically, in the unitary gauge, one encounters divergencies up to the sixth power. It is well known that, in four dimensions, shifting momenta in integrals that are more than logarithmically divergent is a "tricky business"recall the calculation of linearly divergent fermion triangles in chiral anomalies [24, 25]-that requires keeping track of several "surface" terms for these integrals. There is also the situation we face here where apparent logarithmically 


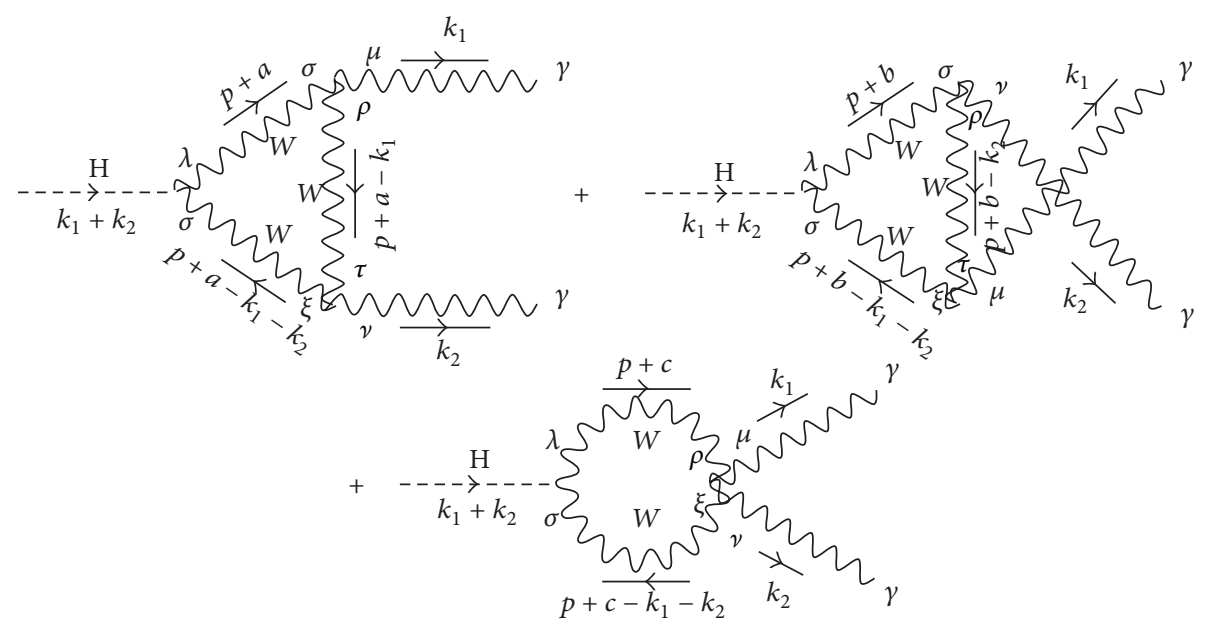

Figure 1: $W$-gauge boson contribution to the $H \rightarrow \gamma \gamma$ amplitude. Momentum flow together with relevant shift vectors is indicated.

divergent integrals turn out to be finite but discontinuous at $d=4$.

We would like to bypass those ambiguities and at the same time to present a "regularisation" method, by performing the calculation for the $H \rightarrow \gamma \gamma$ amplitude strictly in 4 dimensions and in the physical unitary gauge. Our method is similar to the one used elsewhere for calculating triple gauge boson amplitudes $[26,27]$, or Lorentz noninvariant amplitudes [28], and consists of three steps:

(1) We write down the most general Lorentz invariant $H \rightarrow \gamma \gamma$ amplitude.

(2) We introduce arbitrary vectors that account for the "shifting momentum" indeterminacy. We show that a particular choice of those "shifting vectors" cancels higher powers of infinities leaving still behind at most logarithmically divergent integrals that are treated as undetermined variables.

(3) We exploit physics, that is, gauge invariance (Ward Identities) and the GBET, in order to fix the last undetermined variables.

This method is quite general within a renormalizable theory and can be applied to other observables too. Following these steps we arrive at the same result for the $H \rightarrow \gamma \gamma$ amplitude obtained by Ellis et al. [11] and by Shifman et al. [13] almost 35 years ago. Our analysis, among other issues, highlights that the recent observation $[6,7]$ of the $H \rightarrow \gamma \gamma$ at the LHC signifies the validity of the Goldstone Boson Equivalence Theorem. As a further clarification we also make a remark on the direct calculation in the following three cases: we first perform the integrals in exactly $d=4$ (with no regularisation method beyond the one discussed in point 2 above), second, by exploiting Dimensional Regularisation (DR) as defined in $[29,30]$ and then taking the limit $d \rightarrow 4$, and finally third by using a four-dimensional regularization scheme introduced in [31].

Our calculation is complementary to, but somewhat different from, the two existing ones $[19,32,33]$ performed in the unitary gauge. It is not our intend to redo the calculation in unitary gauge with DR as in [19]. On the contrary, we want to clarify subtle issues related to the amplitude in unitary gauge and $d=4$ raised in part by $[32,33]$. We find, using arbitrary vectors, that divergencies (up to the 6th power) are reduced down to logarithmic ones. This is a new result that is not obvious when working in unitary gauge and cannot be seen when using dimensional regularisation. This fact was stated incorrectly in $[32,33]$.

The outline of the paper is as follows: in Section 2 we present the calculation of the $W$-loop contribution (note that the calculation of the fermion triangle contribution is well defined; that is, it is independent of arbitrary vectors and finite. We are not going to repeat this calculation here and refer the reader to the reviews in $[4,5])$ to $H \rightarrow \gamma \gamma$ amplitude, its ambiguities, and the resolution within physics arising from GBET. Next, in Section 3 we examine details of the amplitude calculation within an alternative and recently proposed four-dimensional regularization scheme [31], the one that resembles most closely the symmetry approach taken here. In Section 4 we discuss other possible physical setups plus experiment that may help to resolve inconsistencies. We conclude in Section 5. There are two appendices: Appendix A that contains some intermediate formulae and Appendix B where we present the details about the origin of surface terms in four dimensions.

\section{The $W$-Loop Contribution to $H \rightarrow \gamma \gamma$ in $\mathbf{S M}$}

The most general, Lorentz and CP-invariant, form of the ofshell $H \rightarrow \gamma \gamma$ amplitude is,

$$
\mathscr{M}_{1} g^{\mu \nu}+\mathscr{M}_{2} k_{1}^{\nu} k_{2}^{\mu}+\mathscr{M}_{3} k_{1}^{\mu} k_{2}^{\nu}+\mathscr{M}_{4} k_{1}^{\mu} k_{1}^{\nu}+\mathscr{M}_{5} k_{2}^{\mu} k_{2}^{\nu}
$$

where $k_{1}$ and $k_{2}$ are the outgoing photon momenta shown in Figure 1 and the coefficients $\mathscr{M}_{i=1 \cdots 5} \equiv \mathscr{M}_{i=1 \cdots 5}\left(k_{1}, k_{2}\right)$ are scalar functions of $k_{1}^{2}, k_{2}^{2}$, and $k_{1} \cdot k_{2}$. By considering that all particles are on-mass-shell, that is, $k_{1}^{2}=k_{2}^{2}=0, k_{1} \cdot k_{2}=$ $m_{H}^{2} / 2, k_{1} \cdot \epsilon^{*}\left(k_{1}\right)=0, k_{2} \cdot \epsilon^{*}\left(k_{2}\right)=0$, we obtain an amplitude 
$\mathscr{M}=\mathscr{M}^{\mu \nu} \epsilon_{\mu}^{*}\left(k_{1}\right) \epsilon_{\nu}^{*}\left(k_{2}\right)$ with only two undetermined (for the time being) coefficients

$$
\mathscr{M}^{\mu \nu}=\mathscr{M}_{1} g^{\mu \nu}+\mathscr{M}_{2} k_{1}^{\nu} k_{2}^{\mu} .
$$

In unitary gauge, the Feynman diagrams that contribute to $\mathscr{M}_{1}$ and $\mathscr{M}_{2}$ are displayed in Figure 1 . In order to calculate them, we introduce three arbitrary four-vectors $a, b$, and $c$, one for each diagram. These vectors shift the integration momentum, that is, $p \rightarrow p+a$ for the first diagram, $p \rightarrow$ $p+b$ for the second diagram, and $p \rightarrow p+c$ for the third diagram. As we will see, these arbitrary vectors operate as regulators capable to handle highly divergent integrals related to unitary gauge choice. Furthermore, the vectors $a, b$, and $c$ linearly depend upon the external momenta $k_{1}$ and $k_{2}$. Hence $a, b$, and $c$ are not linearly independent (cf. (4)). This is an important fact leading to the cancellation of infinities.

We first calculate the less divergent part of $\mathscr{M}^{\mu \nu}$ in (2) which is the $\mathscr{M}_{2}$ coefficient. (The coefficient $\mathscr{M}_{1}$ will be fixed later on by the requirement of gauge invariance.) By naive power counting, we see that $\mathscr{M}_{2}$ diverges by at most four powers. Then we perform the Feynman integral calculations strictly in 4 dimensions. For reasons that will become clear later, we shall keep the number of dimensions general in all intermediate steps of the calculation, that is, $g^{\mu \nu} g_{\mu \nu}=d$. As we will see, $d$ contributes only to finite pieces of $\mathscr{M}_{2}$ (cf. (9)). (On the contrary, we shall see that there are nontrivial $d$-contributions into $\mathscr{M}_{1}$-coefficient.)

With all the above definitions, we can write down the total amplitude in the form

$$
\begin{aligned}
\mathscr{M}^{\mu \nu} \sim \int \frac{d^{4} p}{(2 \pi)^{4}}[ & \mathscr{A}_{11} g^{\mu \nu}+\mathscr{A}_{21}(p+a)^{\mu}(p+a)^{\nu} \\
& +\mathscr{A}_{22}(p+b)^{\mu}(p+b)^{\nu} \\
& +\mathscr{A}_{23}(p+c)^{\mu}(p+c)^{\nu} \\
& +\mathscr{A}_{31}(p+a)^{\mu} k_{1}^{\nu}+\mathscr{A}_{32}(p+b)^{\mu} k_{1}^{\nu} \\
& +\mathscr{A}_{33}(p+c)^{\mu} k_{1}^{\nu} \\
& +\mathscr{A}_{41}(p+a)^{\nu} k_{2}^{\mu}+\mathscr{A}_{42}(p+b)^{\nu} k_{2}^{\mu} \\
& \left.+\mathscr{A}_{43}(p+c)^{\nu} k_{2}^{\mu}+\mathscr{A}_{51} k_{2}^{\mu} k_{1}^{\nu}\right],
\end{aligned}
$$

where the coefficients $\mathscr{A}_{i j}=\mathscr{A}_{i j}\left(p^{n} ; k_{1}, k_{2} ; a ; b ; c\right)$ with $-6 \leq$ $n \leq 0$ are given in Appendix A, and the $\sim$ sign is the proportionality factor: $-2 i e^{2} / v$. Note that $\mathscr{M}^{\mu \nu}$ is a (superficially) 6th power divergent amplitude in the unitary gauge. $\mathscr{A}_{11}$ in (3) solely contributes to $\mathscr{M}_{1}$ while all other $\mathscr{A}$-elements contribute to both $\mathscr{M}_{1}$ and/or $\mathscr{M}_{2}$ in (2).

First we focus on the calculation of the "less divergent" coefficient $\mathscr{M}_{2}$ of (2). Based on naive power counting, we observe that the $\mathscr{A}_{21}, \mathscr{A}_{22}, \mathscr{A}_{23}$ terms in (3) lead to and the most quartic divergent integrals. However, when adding all these pieces together, we find that quartic divergent integrals vanish for every arbitrary vectors $a, b$, and $c$ leaving behind an expression with integrals of third power (in momenta) plus integrals with smaller divergencies. Then the cubically divergent integrals are proportional to all possible Lorentz invariant combinations like $[(a+b-2 c) \cdot p] p^{\mu} p^{\nu}$, $\left[(a+b-2 c)^{\nu} p^{\mu}\right] p^{2}$, and $\left[(a+b-2 c)^{\mu} p^{\nu}\right] p^{2}$. Therefore, choosing

$$
a+b-2 c=0
$$

we ensure that third-order divergent integrals related to $\mathscr{A}_{21}, \mathscr{A}_{22}$, and $\mathscr{A}_{23}$ terms vanish identically. In the same way, by naive power counting, $\mathscr{A}_{31}$ and $\mathscr{A}_{33}$ terms - these terms in (3) together with $\mathscr{A}_{32}$ contribute solely to $\mathscr{M}_{2}$-lead again to at most third-order divergent integrals. However, in the sum of $\mathscr{A}_{31}$ and $\mathscr{A}_{33}$ terms in (3), third-order divergent integrals vanish for arbitrary $a, b$, and $c$, leading to an expression that when added to $\mathscr{A}_{32}$ term consists of at most quadratically divergent integrals, proportional to $[(c-a) \cdot p] p^{\mu} k_{1}^{\nu}$ and $\left[(c-a)^{\mu} k_{1}^{\nu}\right] p^{2}$. We choose

$$
c-a=0 \text {, }
$$

for the quadratically divergent integrals to vanish. Likewise, when we add $\mathscr{A}_{42}$ and $\mathscr{A}_{43}$ terms - these terms, together with $\mathscr{A}_{41}$, solely contribute to $\mathscr{M}_{2}$ in (2) - the third-order divergent integrals vanish for every choice of $a, b$, and $c$ leading to an expression that when added to $\mathscr{A}_{41}$ consists of at most quadratically divergent integrals proportional to $[(c-b) \cdot p] p^{\nu} k_{2}^{\mu}$ and $\left[(c-b)^{\nu} k_{2}^{\mu}\right] p^{2}$. Therefore, we choose

$$
c-b=0
$$

for infinities to vanish identically. From (4), (5), and (6) we arrive at the final relation among the three introduced vectors:

$$
a=b=c \text {. }
$$

Equation (7) suggests that the rest of the divergent integrals depend on, at most, one arbitrary vector, say the $a$-vector. Note that $\mathscr{A}_{51}$ contributes only to the finite part of $\mathscr{M}_{2}$. Now, if we impose conditions (7) onto the remaining expressions for $\mathscr{A}_{21}, \mathscr{A}_{22}, \ldots, \mathscr{A}_{51}$ terms of (3), we find that all quadratically and linearly divergent integrals vanish, independently of the direction of the $a$-vector. We stress here on the fact that the cancellation of divergencies down to logarithmic ones is a highly non-trivial, almost "miraculous" result. These cancellations only take place for a particular choice of the momentumvariable shift vectors (7). (As a corollary, if for instance, we had split the $W W \gamma$-vertex into three pieces, each one associated with three different arbitrary vectors, then the generalised condition (7) would again downgrade the divergency of the amplitude to a logarithmic one.) Of course this is an expected outcome for an observable in a renormalizable theory.

Our final result contains at most logarithmically divergent integrals. (This result is different from the one obtained in $[32,33]$, where there are remaining quadratically and linearly divergent terms. Following (11) in Gastmans et al. paper [32] or (3.36) in their sequel paper [33], and 
unless there is a typo in both their formulae, we find that there is a missing quadratically divergent term proportional to $k^{2} k_{\mu} k_{v}$. Even in the case that this is a typo, their formulae contain a linearly divergent term that is also referred to by the authors claiming that this last term reduces to a logarithmically divergent integral by changing the internal momentum $k \rightarrow-k$ and further manipulating the integral. Note here that in our calculation all divergent integrals are reduced to at most logarithmically divergent ones without any further manipulation nor any assumption other than (7) for every arbitrary vector: $a, b$, and $c$. Finally, the authors in $[32,33]$ have made a specific choice for routing the internal loop momentum corresponding to $a=b=$ $c=(1 / 2)\left(k_{1}+k_{2}\right)$, and therefore their result should be the same as ours.) Despite of the fact that the resulting expressions so far contain the shift $p+a$ instead of $p$ with an arbitrary vector $a$, its presence is irrelevant since logarithmically divergent integrals are momentum-variable shift independent [34]. Summing up all the above contributions to $\mathscr{M}_{2}$, we find a particularly nice and symmetric form for $\mathscr{M}^{\mu \nu}$ :

$$
\begin{aligned}
& \mathscr{M}^{\mu \nu} \sim \int \frac{d^{4} p}{(2 \pi)^{4}} p^{\mu} p^{\nu}\left\{\frac{4(d-1) m_{W}^{2}+2 m_{H}^{2}}{\left[p^{2}-m_{W}^{2}\right]\left[\left(p-k_{1}\right)^{2}-m_{W}^{2}\right]\left[\left(p-k_{1}-k_{2}\right)^{2}-m_{W}^{2}\right]}\right. \\
& \left.+\frac{4(d-1) m_{W}^{2}+2 m_{H}^{2}}{\left[p^{2}-m_{W}^{2}\right]\left[\left(p-k_{2}\right)^{2}-m_{W}^{2}\right]\left[\left(p-k_{1}-k_{2}\right)^{2}-m_{W}^{2}\right]}\right\} \\
& +\int \frac{d^{4} p}{(2 \pi)^{4}} p^{\mu} k_{1}^{\nu}\left\{\frac{-4(d-1) m_{W}^{2}-4\left(p \cdot k_{2}\right)}{\left[p^{2}-m_{W}^{2}\right]\left[\left(p-k_{1}\right)^{2}-m_{W}^{2}\right]\left[\left(p-k_{1}-k_{2}\right)^{2}-m_{W}^{2}\right]}\right. \\
& \left.+\frac{-4\left(p \cdot k_{2}\right)}{\left[p^{2}-m_{W}^{2}\right]\left[\left(p-k_{2}\right)^{2}-m_{W}^{2}\right]\left[\left(p-k_{1}-k_{2}\right)^{2}-m_{W}^{2}\right]}\right\} \\
& +\int \frac{d^{4} p}{(2 \pi)^{4}} p^{v} k_{2}^{\mu}\left\{\frac{-4\left(p \cdot k_{1}\right)}{\left[p^{2}-m_{W}^{2}\right]\left[\left(p-k_{1}\right)^{2}-m_{W}^{2}\right]\left[\left(p-k_{1}-k_{2}\right)^{2}-m_{W}^{2}\right]}\right. \\
& \left.+\frac{-4(d-1) m_{W}^{2}-4\left(p \cdot k_{1}\right)}{\left[p^{2}-m_{W}^{2}\right]\left[\left(p-k_{2}\right)^{2}-m_{W}^{2}\right]\left[\left(p-k_{1}-k_{2}\right)^{2}-m_{W}^{2}\right]}\right\} \\
& +\int \frac{d^{4} p}{(2 \pi)^{4}} k_{1}^{\nu} k_{2}^{\mu}\left\{\frac{6 m_{W}^{2}+2 p^{2}}{\left[p^{2}-m_{W}^{2}\right]\left[\left(p-k_{1}\right)^{2}-m_{W}^{2}\right]\left[\left(p-k_{1}-k_{2}\right)^{2}-m_{W}^{2}\right]}\right. \\
& \left.+\frac{6 m_{W}^{2}+2 p^{2}}{\left[p^{2}-m_{W}^{2}\right]\left[\left(p-k_{2}\right)^{2}-m_{W}^{2}\right]\left[\left(p-k_{1}-k_{2}\right)^{2}-m_{W}^{2}\right]}\right\} \text {. }
\end{aligned}
$$

Introducing Feynman parameters, shifting momentum variable from $p$ to $\ell$, and ignoring all terms (these terms will be used later in arriving at (24)) that contribute to $\mathscr{M}_{1}$, we find that the contribution to $\mathscr{M}_{2}$ in (2) arises solely from the term

$$
\begin{aligned}
& \mathscr{M}_{2} k_{1}^{\nu} k_{2}^{\mu} \\
& \sim 8 \int_{0}^{1} d x \int_{0}^{1-x} d y \int \frac{d^{4} \ell}{(2 \pi)^{4}} \\
& \quad \times \frac{\ell^{2} k_{1}^{\nu} k_{2}^{\mu}-2\left(\ell \cdot k_{2}\right) \ell^{\mu} k_{1}^{\nu}-2\left(\ell \cdot k_{1}\right) \ell^{\nu} k_{2}^{\mu}}{\left(\ell^{2}-\Delta\right)^{3}}
\end{aligned}
$$

$$
\begin{aligned}
& +8 m_{W}^{2} \int_{0}^{1} d x \int_{0}^{1-x} d y \\
& \quad \times \int \frac{d^{4} \ell}{(2 \pi)^{4}} \frac{3-2(d-1) x(1-x-y)}{\left(\ell^{2}-\Delta\right)^{3}} k_{1}^{\nu} k_{2}^{\mu}
\end{aligned}
$$

with $\Delta=x(x+y-1) m_{H}^{2}+m_{W}^{2}$. Obviously, the first integral in (9) is (superficially) logarithmically divergent while the second one is finite. The number of dimensions $(d)$ appears only at the finite integral, and therefore we can fearlessly set $d=4$ everywhere. This means that we do not use dimensional regularisation in what follows (see however the discussion 
below). We state here few additional remarks to be exploited later on: (a) we observe that the top line in the integrand of (9) does not vanish in the limit $m_{W}^{2} \rightarrow 0$ and (b) despite of the appearances in (8), there is no $m_{H}^{2}$ in the numerators of the subsequent expression equation (9). The whole $m_{H}^{2}$ contribution arises from the denominator's $\Delta$-term.

Our next step is to parametrize the logarithmically divergent integral in (9) by an unknown, dimensionless parameter $\lambda$ to be determined later by a physical argument. So we define

$$
\begin{aligned}
& \int_{0}^{1} d x \int_{0}^{1-x} d y \int \frac{d^{4} \ell}{(2 \pi)^{4}} \\
& \quad \times \frac{\ell^{2} k_{1}^{\nu} k_{2}^{\mu}-2\left(\ell \cdot k_{2}\right) \ell^{\mu} k_{1}^{\nu}-2\left(\ell \cdot k_{1}\right) \ell^{\nu} k_{2}^{\mu}}{\left(\ell^{2}-\Delta\right)^{3}} \\
& \quad \equiv \frac{i \lambda}{4(4 \pi)^{2}} k_{1}^{\nu} k_{2}^{\mu} .
\end{aligned}
$$

A parenthesis must be added at this point. We could of course promote $d^{4} \ell \rightarrow d^{d} \ell$ and use dimensional regularisation [29] by exploiting symmetric integration $\ell^{\mu} \ell^{\nu} \rightarrow(1 / d) \ell^{2} g^{\mu \nu}$ in $d$-dimensions. In this case, and after taking the limit $d \rightarrow 4$, one finds $\lambda=-1$ which is finite and nonzero and agrees with the one we find below in (20) after imposing the GBET condition. This is also the result found in the original references [11-13]. However, according to [32, 33], the integral in (10) is discontinuous at $d=4$; in fact, when symmetric integration $\ell^{\mu} \ell^{\nu} \rightarrow(1 / 4) \ell^{2} g^{\mu \nu}$ in $d=4$ is used, one finds instead $\lambda=0$. This is also understood in a slightly different context. It has long been known [34-36] that shifts of integration variables in linearly (and above) divergent integrals are accompanied by "surface" terms that appear only in four dimensions, a famous example being the integrals in chiral anomaly triangle graphs. For our purpose here let us start with the following shift of variables in a linearly divergent integral that has been generalised [36] to work in $2 \omega$-dimensions following the expression

$$
\int d^{2 \omega} \ell \frac{\ell_{\mu}}{\left[(\ell-k)^{2}-\Delta\right]^{2}}-\int d^{2 \omega} \ell \frac{(\ell+k)_{\mu}}{\left(\ell^{2}-\Delta\right)^{2}}=-\frac{i \pi^{2}}{2} k_{\mu} \delta_{\omega, 2}
$$

that is valid for $\omega<5 / 2$ and $\Delta$ constant, possibly dependent on Feynman parameters, like the one given below (9), and $k_{\mu}$ is an arbitrary constant four-vector. By taking the derivative, $\partial / \partial k^{\nu}$ of both sides in (11) shifting the integration variable for the logarithmically divergent integral encountered, and evaluating the finite one, we easily arrive at

$$
\int d^{2 \omega} \ell \frac{\ell^{2} g_{\mu \nu}-4 \ell_{\mu} \ell_{v}}{\left(\ell^{2}-\Delta\right)^{3}}=-\frac{i \pi^{2}}{2} g_{\mu \nu}\left(\frac{\pi^{\omega-2} \Gamma(3-\omega)}{\Delta^{2-\omega}}-\delta_{\omega, 2}\right) .
$$

For an alternative and detailed proof of (12), see Appendix B. (The same result is obtained by standard algebraic tricks.
We would like to thank R. Jackiw for communicating his calculation to us.) Applying (12) to $\ell^{\sigma} \ell^{\rho}$ terms of (10) with $d^{4} \ell /(2 \pi)^{4} \rightarrow d^{2 \omega} \ell /(2 \pi)^{2 \omega}$, we find

$$
\lambda= \begin{cases}0, & \omega=2 \\ -1, & \omega=2-\epsilon(\mathrm{DR}) .\end{cases}
$$

This is consistent with the symmetric integration in 4 dimensions $(\omega=2)$ but, is also consistent with the usual tabulated textbook result [37] from dimensional regularisation in 4-2 $\epsilon$ dimensions $(\omega=2-\epsilon)$. Equation (13) shows that $\lambda$ is discontinuous at $d=2 \omega=4$. Then the question arises: which $\lambda$ to believe in? Answer: the one that is indicated by well defined, calculable, boundary conditions and symmetries of the underlying theory.

The above parenthesis to our calculation motivates us to avoid the direct calculation of integral (10), but set $d=$ 4 everywhere and treat $\lambda$ as an unknown parameter to be defined later within a physical context or experiment. Substituting (10) into (9), we arrive at

$$
\begin{aligned}
\mathscr{M}_{2} \sim \frac{i}{8 \pi^{2}}\left\{\lambda-6 m_{W}^{2}\right. \\
\left.\times \int_{0}^{1} d x \int_{0}^{1-x} d y \frac{1-2 x(1-x-y)}{\Delta}\right\} .
\end{aligned}
$$

Evaluating the double finite integral in (14) and restoring the proportionality factor given below (3), we obtain

$$
\mathscr{M}_{2}=-\frac{e^{2} g}{(4 \pi)^{2} m_{W}}\{-2 \lambda+[3 \beta+3 \beta(2-\beta) f(\beta)]\},
$$

where

$$
\begin{aligned}
\beta & =\frac{4 m_{W}^{2}}{m_{H}^{2}}, \\
f(\beta) & = \begin{cases}\arctan ^{2}\left(\frac{1}{\sqrt{\beta-1}}\right), & \beta \geq 1 \\
-\frac{1}{4}\left[\ln \left(\frac{1+\sqrt{1-\beta}}{1-\sqrt{1-\beta}}\right)-i \pi\right]^{2}, & \beta<1 .\end{cases}
\end{aligned}
$$

Our final step is to determine the unknown parameter $\lambda$ in (15). For this we need physics that reproduces $\mathscr{M}_{2}$ in a different and unambiguous way. One choice, probably not the only one, is to adopt the Goldstone Boson Equivalence Theorem (GBET) [20-22] which states that the amplitude for emission or absorption of a longitudinally polarised $W$ at high energy becomes equivalent to the emission or absorption of the Goldstone boson that was eaten. Mathematically, this is written by an [38]

$$
S\left[W_{L}^{ \pm}, \text {physical }\right]=i^{n} S\left[s^{ \pm}, \text {physical }\right],
$$

which says that the $S$-matrix elements for the scattering of the physical longitudinal vector bosons $W_{L}$ with other physical particles are the same as the $S$-matrix elements 

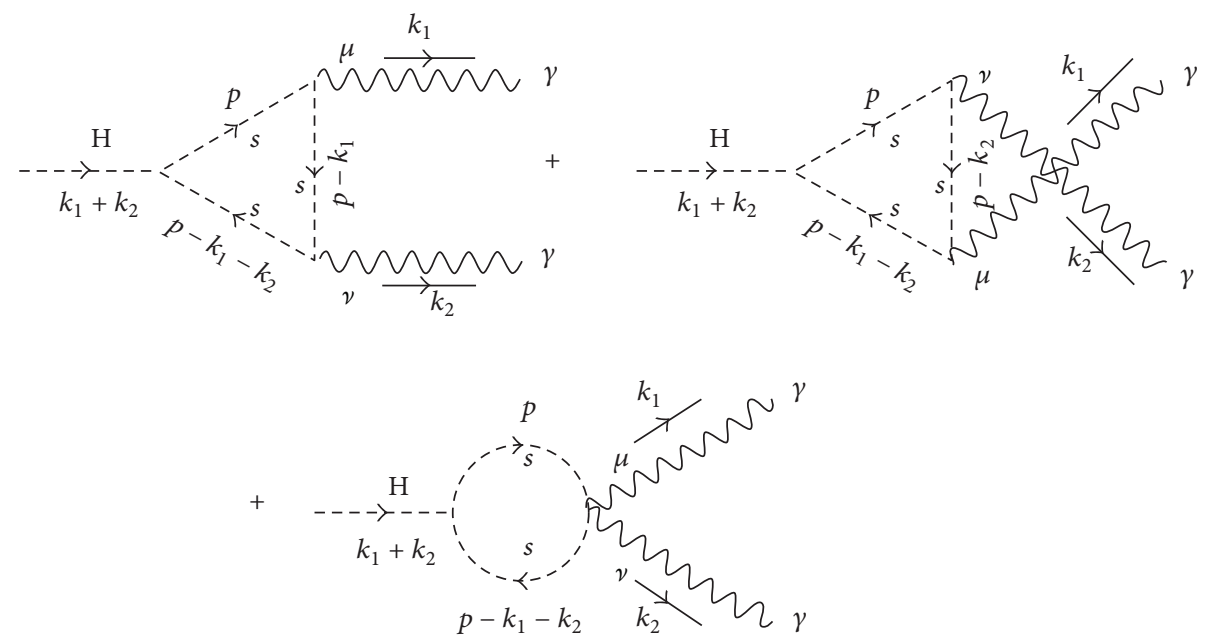

Figure 2: Charged Goldstone boson contributions to $H \rightarrow \gamma \gamma$ in the limit of $g \rightarrow 0$.

of the theory where the $W_{L}$ 's have been replaced by physical Goldstone bosons $\left(s^{ \pm}\right)$. We are not going to get into details here; apart from the original literature the reader is also referred to the articles [19, 38-40]. Following [38], within perturbation theory and in the limit of high energies, $m_{W}^{2} / s \rightarrow 0$, GBET can be expressed with physics in two different limits of the theory: (a) $g^{2} / \lambda_{H} \rightarrow 0$ or (b) $m_{H}^{2} / s \rightarrow 0$.

The limit (b) is irrelevant (the limit (b) simply says that matrix elements for the theory which contains the physical $W_{L}$ 's and zero v.e.v are equal to those produced by scattering of massless physical Goldstone bosons (instead of $W_{L}$ 's) at high energies. We have checked that (17) is satisfied in this limit) for defining $\lambda$ in (15) so we completely focus on the limit (a). It is very easy to see that, in the unitary gauge, the $W_{L}$ 's do not decouple (this is another advantage of calculating in the unitary gauge. Note that here the word "decoupling" is stated in a different context than is usually quoted following the Appelquist-Carazzone [41] theorem, where particle masses circulating in loops are much heavier than the external ones) for vanishing gauge coupling $g$. Consider for example the diagrams in Figure 1: there is always an $m_{W}^{2}$ from the $H W W$-vertex that cancels another $m_{W}^{2}$ sitting in the denominator of the longitudinal part for the internal $W$ boson propagator expression written in the unitary gauge. So, as it was already noted in the paragraph below (9), in the limit $g \rightarrow 0$, there are remaining nondecoupled terms. Unfortunately, these effects may be obscured or misjudged by the regularisation method needed to handle divergent, intermediate loop integrals. This is exactly what happens here when trying to calculate $\lambda$ directly from its ambiguous form (10). On the other hand, however, at the exact $g=0$, with fixed v.e.v $v$ and Higgs quartic coupling $\lambda_{H}$, (17) suggests that the Goldstone bosons $\left(s^{ \pm}\right)$should reappear at the physical spectrum of the theory while the longitudinal components of $W$ 's become unphysical. At this limit, the SM is a spontaneously broken global $S U(2)_{L} \times U(1)_{Y}$-symmetry that couples, minimally, to electromagnetism. The interactions between the Higgs and photon with the Goldstone bosons are simply those of a spontaneously broken scalar QED with $U(1)_{\mathrm{em}}$ :

$$
\begin{gathered}
H s^{+} s^{-}:-\frac{i m_{H}^{2}}{v}, \\
\gamma s^{+}\left(p_{1}\right) s^{-}\left(p_{2}\right):-i e\left(p_{1}+p_{2}\right)^{\mu}, \\
\gamma \gamma s^{+} s^{-}: 2 i e^{2} g^{\mu \nu} .
\end{gathered}
$$

Armed with these Feynman rules we calculate the diagrams in Figure 2. By doing so, we introduce again three momentum variable shift vectors, one for each diagram, exactly in the same way we did for the calculation of the diagrams in Figure 1. The Lorentz structure of the amplitude is completely analogous to (2) with $\mathscr{M}_{1,2} \rightarrow \mathscr{M}_{1,2 \text { (GBET) }}$, but now due to the scalar propagators, the superficial degree of divergence, for diagrams contributing to $\mathscr{M}_{2 \text { (GBET) }}$, is $D=-2$. Hence, all integrals involved in $\mathscr{M}_{2 \text { (GBET) }}$ are finite and in addition, they are independent of any momentum integration shift vector variable. As a consequence, $\mathscr{M}_{2 \text { (GBET) }}$ is well defined, calculable, independent of any regularisation method, and at the limit of $g \rightarrow 0\left(\right.$ or $\beta=4 m_{W}^{2} / m_{H}^{2} \rightarrow 0$ ) is

$$
\mathscr{M}_{2(\mathrm{GBET})}=-\frac{2 e^{2} g}{(4 \pi)^{2} m_{W}}, \quad \beta \longrightarrow 0 .
$$

By equating (15) (in the limit $\beta \rightarrow 0$ ) and (19) which represent the 1.h.s and r.h.s of the GBET condition (17), respectively, we find

$$
\lambda=-1
$$

This value agrees with dimensional regularization $[29,30]$ in the limit $d \rightarrow 4$ (see (13)). The final form of the $\mathscr{M}_{2}$ in (2) is

$$
\mathscr{M}_{2}=-\frac{e^{2} g}{(4 \pi)^{2} m_{W}}\{2+[3 \beta+3 \beta(2-\beta) f(\beta)]\},
$$

with $\beta$ and $f(\beta)$ defined in (16). 
To complete the picture there is still the coefficient $\mathscr{M}_{1}$ in (2) to be calculated. Naive power counting says that this is by two powers more divergent than $\mathscr{M}_{2}$ and, in general, undetermined. It can be fixed however by using quantum gauge invariance, that is, conservation of charge, for the $U(1)_{\mathrm{em}}$ :

$$
\begin{aligned}
& k_{1 \mu} \mathscr{M}^{\mu \nu}=0, \\
& k_{2 v} \mathscr{M}^{\mu \nu}=0, \\
& k_{1}^{2}=k_{2}^{2}=0,
\end{aligned}
$$

and thus from (2),

$$
\mathscr{M}_{1}=-\left(k_{1} \cdot k_{2}\right) \mathscr{M}_{2}
$$

Equation (23) is substituted to (2) with $\mathscr{M}_{2}$ read by (21). This is exactly the same result for the $W$-boson contribution to $H \rightarrow \gamma \gamma$ amplitude that has been obtained in $[11-14,19]$ using dimensional regularisation in $R_{\xi}$-gauges.

It is interesting here to note the result from the explicit algebraic manipulation of $\mathscr{M}_{1}$ in the unitary gauge and check the validity of gauge invariance (see (23)). Exactly as for $\mathscr{M}_{2}$, the condition $a=b=c$ for the arbitrary vectors given in (7) is crucial in reducing the divergence of $\mathscr{M}_{1}$ down to a logarithmic one (see expression equation (A.2)). In $d$ dimensions the expression for $\mathscr{M}_{1}$ is finally independent of any arbitrary vector and, up to a proportionality factor, reads

$$
\begin{aligned}
\mathscr{M}_{1} \sim 4 \int_{0}^{1} d x \int_{0}^{1-x} d y \int \frac{d^{d} \ell}{(2 \pi)^{d}}\{ & \frac{4\left(\ell \cdot k_{1}\right)\left(\ell \cdot k_{2}\right)+2(2 / d-1) \ell^{2}\left(k_{1} \cdot k_{2}\right)+((d-1) / d)(4-d) \ell^{2} m_{W}^{2}}{\left(\ell^{2}-\Delta\right)^{3}}+ \\
& \left.+\frac{(d-1) m_{W}^{4}-3 m_{W}^{2} m_{H}^{2}+(1-d) x(x+y-1) m_{W}^{2} m_{H}^{2}}{\left(\ell^{2}-\Delta\right)^{3}}\right\} .
\end{aligned}
$$

Clearly the first integral in (24) is ill-defined in four dimensions. If, however, we insist on doing the calculation of (24) in $d=4$ with symmetric integration, like in $[32,33]$, we find that gauge invariance (see (23)) is not satisfied. This is of course unacceptable. By going a little bit deeper, gauge invariance is lost because of the term proportional to $4-d$ in (24) which vanishes when $d=4$. Quite the contrary in DR, this term results in a non-zero contribution when $m_{W} \neq 0$ since the (log divergent) integral in front of $(4-d)$ contains a simple pole at $d=4$. This changes the final result and renders (21), (24), and (23) consistent only if $\lambda=-1$. This outcome is in agreement with [19].

Few remarks are worth mentioning here. Had we started first calculating $\mathscr{M}_{1}$, there would be no possibility of defining unambiguously $\lambda$ without using a gauge invariant regulator: the $g_{\mu \nu}$ part of the amplitude at $g \rightarrow 0$ involving Goldstone bosons (see diagrams Figure 2) is not well defined-an integral as the one in (12) appears again. Another remark is that the same expressions for the coefficients $\mathscr{A}_{i j}$ displayed in Appendix A in the unitary gauge appear also when one exploits the $R_{\xi}$-gauge. In the latter there are in addition $\xi$ dependent terms [19] that vanish in the end from unphysical scalar contributions. Therefore, the logarithmic ambiguity in (12), found here in the unitary gauge, is similar in every other gauge.

The ambiguity of the integral in (12) has been discussed by many articles in the recent literature. References [15, 16] have used gauge-invariant regulators alá Pauli-Villars. Most notably, Piccinini et al. [18] showed that the unitary gauge with a cut-off regularisation scheme turns out to be nonpredictive: new physics input, along the lines of [28] also followed in our article, is needed. As already noted, Marciano et al. [19] were the first to make the calculation of $h \rightarrow \gamma \gamma$ in unitary gauge with DR.
Furthermore, $[15,18,19,39,40]$ showed that the "decoupling limit" $m_{W} / m_{H} \rightarrow 0$ must hold because of the GBET. All these articles together with the one at hand conclude that the result in $[32,33]$ is incorrect. Furthermore, the aim of our paper is not to redo the calculation in the unitary gauge with DR as in [19]; this is only a byproduct of our analysis. On the contrary, we want to clarify subtle issues related to this calculation in the unitary gauge and in $d=4$ raised in part by $[32,33]$. We find by working strictly in $d=4$, using arbitrary vectors, that divergencies (up to the 6th power) are reduced down to logarithmic ones. This is a new result that is not obvious when working in the unitary gauge and cannot be seen when using dimensional regularisation.

\section{Four-Dimensional Regularization (FDR)}

So far we have proposed a regularization scheme which is four-dimensional and uses the basic symmetries and underlying physics of the SM. However, in more complicated models or observables with more parameters to adjust, such a scheme can become cumbersome. For example, it is not always obvious which physics argument will fix undefined integrals.

Very recently, Pittau [31] proposed a scheme that is fairly easy to handle and, to the best of our knowledge, is the closest to four-dimensional calculations and thereby coined four-dimensional regularisation/renormalization scheme or just FDR. According to this scheme, infinite bubble graph contributions, that is, large loop momenta contributions that do not depend upon external momenta, are absorbed into the shift of the vacuum while the remaining finite corrections are calculable in four dimensions in addition to being Lorentz and gauge invariant.

We have applied FDR to the calculation of the $H \rightarrow \gamma \gamma$ amplitude and found agreement with our physics approach 
and with DR results. In FDR one introduces an arbitrary scale $\mu$ which is considered to be much smaller than internal momenta and particle masses in loops. Self-contracted loop momenta quantities like $\ell^{2}$ become $\bar{\ell}^{2}=\ell^{2}-\mu^{2}$, while, for gauge invariance to hold, vector momenta, $p^{\mu}$, remain untouched. For example the integral of (12) becomes

$$
\int\left[d^{4} \ell\right] \frac{\bar{\ell}^{2} g_{\mu \nu}-4 \ell_{\mu} \ell_{\nu}}{\bar{D}^{3}}=\int\left[d^{4} \ell\right] \frac{-\mu^{2}}{\bar{D}^{3}} g_{\mu \nu}
$$

where $\bar{D}=\left(\bar{\ell}^{2}-\Delta\right)$ and $\left[d^{4} \ell\right]$ stands for integration over $d^{4} \ell$, dropping all divergent terms from the integrand (see below) and taking the limit $\mu \rightarrow 0$. In going from l.h.s to r.h.s of (25) the symmetry property $\ell_{\mu} \ell_{\nu}=g_{\mu \nu} \ell^{2} / 4$ has been used in four dimensions. Then, using the partial fractions identity,

$$
\frac{1}{\bar{D}^{3}}=\left[\frac{1}{\bar{\ell}^{6}}\right]+\Delta\left(\frac{1}{\bar{D}^{3} \bar{\ell}^{2}}+\frac{1}{\bar{D}^{2} \bar{\ell}^{4}}+\frac{1}{\overline{D \ell}^{6}}\right),
$$

the term in square bracket is recognised as divergent and therefore removed, and integrating the r.h.s of (25) over $\left[d^{4} \ell\right]$ one obtains

$$
\begin{aligned}
& \int\left[d^{4} \ell\right] \frac{-\mu^{2}}{\bar{D}^{3}} \\
& \equiv-\Delta \lim _{\mu \rightarrow 0} \mu^{2} \int d^{4} \ell\left(\frac{1}{\bar{D}^{3} \bar{\ell}^{2}}+\frac{1}{\bar{D}^{2} \bar{\ell}^{4}}+\frac{1}{\bar{D} \bar{\ell}^{6}}\right) \\
& =-\frac{i \pi^{2}}{2},
\end{aligned}
$$

that is, exactly the same result as in DR which eventually leads to $\lambda=-1$ consistent with gauge invariance and GBET. What in fact FDR scheme does is to restate the correct DR answer through the regulator $\mu^{2}$ keeping (12) correct in $d=4$. We therefore understand that the constant ( $\beta$-independent) term of (21) in FDR arises from the fact that the arbitrary scale, $\mu^{2}$, must disappear from physical observables.

\section{Discussion}

It is evident that our calculation for the amplitude incorporates two physical inputs: one is the conservation of charge and the other is the equivalence theorem. They are both direct consequences of the gauge invariance of the underlying physical theory. The first one is experimentally indisputable while the second one is theoretical (this is not entirely correct. There is of course the high energy behaviour of $e^{+} e^{-} \rightarrow$ $W^{+} W^{-}$found at LEP [42] consistent with the GBET.) and has been proven in [43], which is valid in any spontaneously broken renormalizable theory, like, for example, the SM. One may think however that there is a loophole in our use of this second argument: so far, and, to our knowledge, the replacement of the $W$-bosons with Goldstone bosons at high energies has been proven to be valid only for external $W$ bosons $[38,44,45]$ and not for internal ones which is the case exploited here. Although it has been tested in several phenomenological examples [46], a formal, to all orders, proof is still missing. Although this may be true, it is difficult to argue against the validity of decoupling limit $g \rightarrow 0$ (with fixed v.e.v and Higgs quartic coupling) discussed in the paragraph above (18).

Is there another physics context from which one can define $\lambda$ ? One possibility is to exploit the low-energy Higgs theorem [11, 47-49] instead. Although this may serve as a consistency check and indeed is compatible with $\lambda=-1$, we cannot use it to define $\lambda$. The reason here is threefold: first, when treating the Higgs field as an external background field with zero momentum one needs to take partial derivative w.r.t $m_{W}$ of the 2-point photon vacuum polarization amplitude, $\Pi_{\gamma \gamma}\left(q^{2}\right)$. The latter is notoriously difficult, if meaningful, to be calculated in the unitary gauge. Second, according to [11], we know that to the lowest order in weak coupling, the amplitude for the process $\langle\gamma \gamma \mid H\rangle$ is proportional to $\left\langle\gamma \gamma\left|\Theta_{\mu}^{\mu}\right| 0\right\rangle$, where $\Theta_{\mu}^{\mu}=2 m_{W}^{2} W^{+} W^{-}+\cdots$ is the improved energy momentum tensor [50]. However, the calculation of $\left\langle\gamma \gamma\left|\Theta_{\mu}^{\mu}\right| 0\right\rangle$ goes through the same steps as for the calculation for the $H \rightarrow$ $\gamma \gamma$ amplitude and therefore involves the same ambiguity for calculating $\lambda$. Third, one could examine the $W$-contribution to $H \rightarrow \gamma \gamma$ within the dispersion relation approach. It can be shown [51] that the nonvanishing limit at $g_{W} \rightarrow 0$ is due to a finite subtraction induced by the corresponding trace anomaly [52]. However, in order to calculate unambiguously this finite piece, one has to make full use of a (physical) boundary condition of the theory.

As a final remark, suppose that we did not know DR and wanted to calculate a certain observable in 4 dimensions. In this observable we encounter singularities, that is, undefined and undetermined integrals. Then we use physics arguments to fix these ambiguities. However, we can always question whether we are using the right physics setup or not. In that sense the final judgement should come from the experiment. Therefore it may be not academic to ask whether LHC could see the difference between $\lambda=-1$ and $\lambda=0$. Setting the SM Higgs mass $m_{H}=125 \mathrm{GeV}$ and including the top-loop contribution, we find

$$
\frac{\mathrm{Br}(H \longrightarrow \gamma \gamma, \lambda=0)}{\mathrm{Br}(H \longrightarrow \gamma \gamma, \lambda=-1)} \approx 0.46 .
$$

This is certainly within LHC's sensitivity for $14 \mathrm{TeV}$ c.m energy and luminosity of $30 \mathrm{fb}^{-1}$ (see, e.g., Figure 3 in [53]). In fact, the recent observation by LHC experiments $[6,7]$ indicates a value $\operatorname{Br}(H \rightarrow \gamma \gamma,(\exp )) / \operatorname{Br}(H \rightarrow \gamma \gamma, \lambda=$ $-1)=1.6 \pm 0.3[54]$ which highly disfavours the case $\lambda=0$ by almost four standard deviations. We can turn this around and state that this is an indirect hint towards the validity of the equivalence theorem.

\section{Conclusions}

In this work we review the $W$-gauge boson loop contribution to the $H \rightarrow \gamma \gamma$ amplitude in the unitary gauge. Our objective is to fix intermediate step indeterminacies arising from divergent diagrams by making full use of physics at $d=4$ much in the same way as in the calculation of the chiral anomaly triangle. 
We anticipate a finite result for the loop-induced $H \rightarrow$ $\gamma \gamma$-amplitude in the renormalizable SM. Therefore the amplitude has to be independent of any shifting momentum variables we have originally introduced. But finite or even log divergent integrals are independent of these vectors, so the vectors have to be accompanied only by infinite contributions, if at all. Therefore, infinities and arbitrary vectors are eliminated altogether by a certain combination among them (see (7)).

The whole calculation in the unitary gauge boils down to a logarithmically divergent integral (10). We find that this integral results in two different values depending on whether $d \rightarrow 4$ or $d=4$. This is due to a surface term remaining at the exact $d=4$ case after the part-by-part integration in $d$-dimensions (see Appendix B). To proceed, we identify this integral with an undefined parameter $\lambda$ (see (12)). This parameter is then fixed unambiguously by assuming the validity of the Goldstone Boson Equivalence Theorem (GBET). Its value is consistent with DR in the limit $d \rightarrow 4$.

In our calculation we are very careful not to perform shifting of integration variables for highly divergent integrals by introducing three arbitrary momentum variable shift vectors straight from the beginning. Divergencies and arbitrariness from these unknown vectors are altogether removed, leaving behind a log-like divergent integral in $\mathscr{M}_{2}$ of (2). This is defined by a physical input taken from the GBET and is connected to $\mathscr{M}_{1}$ by electromagnetic charge conservation.

As noted many times in the text, the key point towards deriving an unambiguous amplitude for $H \rightarrow \gamma \gamma$ in the unitary gauge is the limit of vanishing gauge couplings; this is an aspect of GBET (see (17)). In this limit, the Goldstone boson loop contributions to the coefficient $\mathscr{M}_{2}$ finite, that is, independent of any regularisation scheme.

We also saw that DR (FDR), a regularisation scheme introduced to maintain Ward Identities at intermediate steps of a calculation, supports the GBET in the limit $d \rightarrow 4$ $(d=4)$. On the contrary, we find that performing the integrals in $d=4$ with symmetric integration is not a good choice because it leads to the violation of gauge invariance (see (23) and the discussion below). The main reason is due to surface terms that are developed in exactly $d=4$ dimensions (see discussion below (10) and Appendix B). The latter are axiomatically discarded in $\operatorname{DR}[29,30]$. Another reason is the appearance of the $(d-4)$-term in the numerator of (24).

In conclusion, the four-dimensional calculation of $H \rightarrow$ $\gamma \gamma$ amplitude in the unitary gauge is ambiguous without introduction of a physics input beyond gauge invariance. As we have demonstrated, this physics, which uniquely defines the amplitude, may arise from the Goldstone Boson Equivalence Theorem (GBET). This effectively proves that GBET comprises an additional important pillar of the Standard Model dynamics.

\section{Appendices}

\section{A. The Coefficients $\mathscr{A}_{i j}$}

We append here the integrand expressions for the coefficients $\mathscr{A}_{i j}$ in (3). The corresponding formula for $\mathscr{A}_{11}$ is quite long and is not included here. It can be provided by the authors upon request. Note that the number of dimensions $d$ has been kept arbitrary throughout, and on-shell conditions for the external particles have been imposed:

$$
\begin{aligned}
& \mathscr{A}_{21}=1 \times\left(\left[(p+a)^{2}-m_{W}^{2}\right]\left[\left(p+a-k_{1}\right)^{2}-m_{W}^{2}\right]\right. \\
& \left.\times\left[\left(p+a-k_{1}-k_{2}\right)^{2}-m_{W}^{2}\right]\right)^{-1} \\
& \times\left\{(4 d-6) m_{W}^{2}\right. \\
& +\left[3(p+a) \cdot(p+a)-5(p+a) \cdot k_{1}\right. \\
& \left.-(p+a) \cdot k_{2}+2 m_{H}^{2}\right] \\
& +\frac{1}{m_{W}^{2}}\left[-((p+a) \cdot(p+a))^{2}+3\left((p+a) \cdot k_{1}\right)\right. \\
& \times((p+a) \cdot(p+a))-2\left((p+a) \cdot k_{1}\right)^{2} \\
& -2\left((p+a) \cdot k_{1}\right)\left((p+a) \cdot k_{2}\right) \\
& \left.\left.+((p+a) \cdot(p+a))\left((p+a) \cdot k_{2}\right)\right]\right\}, \\
& \mathscr{A}_{22}=1 \times\left(\left[(p+b)^{2}-m_{W}^{2}\right]\left[\left(p+b-k_{2}\right)^{2}-m_{W}^{2}\right]\right. \\
& \left.\times\left[\left(p+b-k_{1}-k_{2}\right)^{2}-m_{W}^{2}\right]\right)^{-1} \\
& \times\left\{(4 d-6) m_{W}^{2}+\left[3(p+b) \cdot(p+b)-5(p+b) \cdot k_{2}\right.\right. \\
& \left.-(p+b) \cdot k_{1}+2 m_{H}^{2}\right] \\
& +\frac{1}{m_{W}^{2}}\left[-((p+b) \cdot(p+b))^{2}+3\left((p+b) \cdot k_{2}\right)\right. \\
& \times((p+b) \cdot(p+b))-2\left((p+b) \cdot k_{2}\right)^{2} \\
& -2\left((p+b) \cdot k_{1}\right)\left((p+a) \cdot k_{2}\right) \\
& \left.\left.+((p+b) \cdot(p+b))\left((p+b) \cdot k_{1}\right)\right]\right\}, \\
& \mathscr{A}_{23}=\frac{-1}{\left[(p+c)^{2}-m_{W}^{2}\right]\left[\left(p+c-k_{1}-k_{2}\right)^{2}-m_{W}^{2}\right]} \\
& \times\left\{4+\frac{2}{m_{W}^{2}}[-((p+c) \cdot(p+c))\right. \\
& \left.\left.+(p+c) \cdot k_{1}+(p+c) \cdot k_{2}\right]\right\}, \\
& \mathscr{A}_{31}=1 \times\left(\left[(p+a)^{2}-m_{W}^{2}\right]\left[\left(p+a-k_{1}\right)^{2}-m_{W}^{2}\right]\right. \\
& \left.\times\left[\left(p+a-k_{1}-k_{2}\right)^{2}-m_{W}^{2}\right]\right)^{-1} \\
& \times\left\{(7-4 d) m_{W}^{2}-[4(p+a) \cdot(p+a)-7(p+a)\right. \\
& \left.\cdot k_{1}+3(p+a) \cdot k_{2}\right] \\
& +\frac{1}{m_{W}^{2}}\left[((p+a) \cdot(p+a))^{2}\right.
\end{aligned}
$$




$$
\begin{aligned}
& -3\left((p+a) \cdot k_{1}\right)((p+a) \cdot(p+a)) \\
& +2\left((p+a) \cdot k_{1}\right)^{2}+2\left((p+a) \cdot k_{1}\right) \\
& \times\left((p+a) \cdot k_{2}\right)-((p+a) \cdot(p+a)) \\
& \left.\left.\times\left((p+a) \cdot k_{2}\right)\right]\right\} \text {, } \\
& \mathscr{A}_{32}=-1 \times\left(\left[(p+b)^{2}-m_{W}^{2}\right]\left[\left(p+b-k_{2}\right)^{2}-m_{W}^{2}\right]\right. \\
& \left.\times\left[\left(p+b-k_{1}-k_{2}\right)^{2}-m_{W}^{2}\right]\right)^{-1} \\
& \times\left\{m_{W}^{2}+\left[-(p+b) \cdot(p+b)+6(p+b) \cdot k_{2}\right]\right\}, \\
& \mathscr{A}_{33}=\frac{1}{\left[(p+c)^{2}-m_{W}^{2}\right]\left[\left(p+c-k_{1}-k_{2}\right)^{2}-m_{W}^{2}\right]} \\
& \times\left\{2-\frac{1}{m_{W}^{2}}[(p+c) \cdot(p+c)\right. \\
& \left.\left.-(p+c) \cdot k_{1}-(p+c) \cdot k_{2}\right]\right\} \\
& \mathscr{A}_{41}=-1 \times\left(\left[(p+a)^{2}-m_{W}^{2}\right]\left[\left(p+a-k_{1}\right)^{2}-m_{W}^{2}\right]\right. \\
& \left.\times\left[\left(p+a-k_{1}-k_{2}\right)^{2}-m_{W}^{2}\right]\right)^{-1} \\
& \times\left\{m_{W}^{2}+\left[-(p+a) \cdot(p+a)+6(p+a) \cdot k_{1}\right]\right\}, \\
& \mathscr{A}_{42}=1 \times\left(\left[(p+b)^{2}-m_{W}^{2}\right]\left[\left(p+b-k_{2}\right)^{2}-m_{W}^{2}\right]\right. \\
& \left.\times\left[\left(p+b-k_{1}-k_{2}\right)^{2}-m_{W}^{2}\right]\right)^{-1} \\
& \times\left\{(7-4 d) m_{W}^{2}-[4(p+b) \cdot(p+b)\right. \\
& \left.-7(p+b) \cdot k_{2}+3(p+b) \cdot k_{1}\right] \\
& +\frac{1}{m_{W}^{2}}\left[((p+b) \cdot(p+b))^{2}\right. \\
& -3\left((p+b) \cdot k_{2}\right)((p+b) \cdot(p+b)) \\
& +2\left((p+b) \cdot k_{2}\right)^{2}+2\left((p+b) \cdot k_{1}\right) \\
& \times\left((p+b) \cdot k_{2}\right)-((p+b) \cdot(p+b)) \\
& \left.\left.\times\left((p+b) \cdot k_{1}\right)\right]\right\}, \\
& \mathscr{A}_{43}=\mathscr{A}_{33} \text {, } \\
& \mathscr{A}_{51}=1 \times\left(\left[(p+a)^{2}-m_{W}^{2}\right]\left[\left(p+a-k_{1}\right)^{2}-m_{W}^{2}\right]\right. \\
& \left.\times\left[\left(p+a-k_{1}-k_{2}\right)^{2}-m_{W}^{2}\right]\right)^{-1} \\
& \times\left\{5 m_{W}^{2}+\left[3(p+a) \cdot(p+a)-2(p+a) \cdot k_{1}\right]\right\} \\
& +1 \times\left(\left[(p+b)^{2}-m_{W}^{2}\right]\left[\left(p+b-k_{2}\right)^{2}-m_{W}^{2}\right]\right. \\
& \left.\times\left[\left(p+b-k_{1}-k_{2}\right)^{2}-m_{W}^{2}\right]\right)^{-1}
\end{aligned}
$$

$$
\begin{gathered}
\times\left\{5 m_{W}^{2}+\left[3(p+b) \cdot(p+b)-2(p+b) \cdot k_{2}\right]\right\} \\
-2 \times\left(\left[(p+c)^{2}-m_{W}^{2}\right]\right. \\
\left.\times\left[\left(p+c-k_{1}-k_{2}\right)^{2}-m_{W}^{2}\right]\right)^{-1} .
\end{gathered}
$$

It is straightforward, but long and tedious, to show that after implementing the condition (7) to coefficients in (A.1) we arrive at (8) which is at most logarithmically divergent.

For complementarity reasons, it is useful in deriving (24) to present the expression for the coefficient $\mathscr{A}_{11}$ after the imposition of the arbitrary vector relation equation (7):

$$
\begin{gathered}
\mathscr{A}_{11}=1 \times\left(\left[(p+a)^{2}-m_{W}^{2}\right]\left[\left(p+a-k_{1}\right)^{2}-m_{W}^{2}\right]\right. \\
\left.\times\left[\left(p+a-k_{1}-k_{2}\right)^{2}-m_{W}^{2}\right]\right)^{-1} \\
\times\left\{\left(\left(p+a-k_{1}\right)^{2}-m_{W}^{2}\right)(1-d) m_{W}^{2}\right. \\
+4\left[(p+a) \cdot k_{1}\right]\left[(p+a) \cdot k_{2}\right] \\
\left.-\left[3 m_{W}^{2}+(p+a)^{2}\right] m_{H}^{2}\right\} \\
+1 \times\left(\left[(p+a)^{2}-m_{W}^{2}\right]\left[\left(p+a-k_{2}\right)^{2}-m_{W}^{2}\right]\right. \\
\left.\quad \times\left[\left(p+a-k_{1}-k_{2}\right)^{2}-m_{W}^{2}\right]\right)^{-1} \\
\times\{( \\
\left.\left.+4+a-k_{2}\right)^{2}-m_{W}^{2}\right)(1-d) m_{W}^{2} \\
\left.+4(p+a) \cdot k_{1}\right]\left[(p+a) \cdot k_{2}\right] \\
\left.\quad\left[3 m_{W}^{2}+(p+a)^{2}\right] m_{H}^{2}\right\} .
\end{gathered}
$$

This integrand expression, under $\int d^{4} p$, is obviously at most logarithmically divergent.

\section{B. Dimensional Regularization and the Surface Term}

We would like to examine the surface terms arising in $d=4$ when calculating the integral on the l.h.s of (12). This integral after Wick rotation into Euclidean space reads

$$
i \int d^{2 \omega} \ell \frac{\ell^{2} g_{\mu \nu}-4 \ell_{\mu} \ell_{\nu}}{\left(\ell^{2}+\Delta\right)^{3}}
$$

where $\ell \equiv \ell_{E}$ and drop for clarity the subscript $E$ from now on. We follow very closely 't Hooft and Veltman's seminal paper in [29]. In our calculation for a physical process we should notice first that $\ell_{\mu}, \ell_{\nu}$ are strictly 4 -vectors since they are contracted with physical external momenta $k_{1,2}^{\mu}$ or $k_{1,2}^{v}$. On the other hand, the loop momentum $\ell$ in $\ell^{2}$ has components in all, $d=2 \omega$, dimensions. We write $\ell$ as a sum of a vector $\ell_{\|}$which has non-zero components in dimensions 
$0,1,2$, and 3 and a vector $\ell_{\perp}$ which has nonzero components in $(2 \omega-4)$-dimensions:

$$
\ell=\ell_{\|}+\ell_{\perp}
$$

With this definition, the integral (B.1) reduces to

$$
i \int d^{2 \omega} \ell \frac{\ell_{\perp}^{2} g_{\mu \nu}}{\left(\ell^{2}+\Delta\right)^{3}}
$$

where the $\ell_{\|}$components in the numerator of (B.1) vanish thanks to symmetric integration formula, $\ell_{\|}^{\mu} \ell_{\|}^{\nu} \rightarrow$ $(1 / 4) \ell_{\|}^{2} g^{\mu \nu}$. In order not to carry the $g_{\mu \nu}$ in all formulae below we just concentrate on the integral

$$
\begin{aligned}
\mathscr{I} & \equiv i \int d^{2 \omega} \ell \frac{\ell_{\perp}^{2}}{\left(\ell^{2}+\Delta\right)^{3}} \\
& =i \int d^{4} \ell_{\|} \int d^{2 \omega-4} \ell_{\perp} \frac{\ell_{\perp}^{2}}{\left(\ell_{\|}^{2}+\ell_{\perp}^{2}+\Delta\right)^{3}} .
\end{aligned}
$$

Integrating over the extra-dimensional solid angle $d \Omega_{2 \omega-4}$ we arrive at

$$
\mathscr{I}=\frac{2 i \pi^{\omega-2}}{\Gamma(\omega-2)} \int d^{4} \ell_{\|} \int_{0}^{\infty} d L \frac{L^{2 \omega-3}}{\left(\ell_{\|}^{2}+L^{2}+\Delta\right)^{3}},
$$

where $\Gamma(x)$ is the Euler $\Gamma$-function and $L$ is the length of the $\ell_{\perp}$ vector. This integral is UV divergent for $\omega \geq 2$ and IR divergent for $\omega \leq 1$. Therefore, the region of convergence, $1<\omega<2$, is finite, but it does not yet include the point $\omega=2$. In order to enlarge the region of convergence to include $\omega=2$ one has to analytically continue $\mathscr{I}$ by inserting the identity

$$
1=\frac{1}{5}\left(\frac{\partial \ell_{\| \mu}}{\partial \ell_{\| \mu}}+\frac{\partial L}{\partial L}\right)
$$

in (B.5). After integrating by parts in the region of convergence, rewriting the r.h.s in terms of $\mathscr{I}$ from (B.5), and keeping only, potentially, nonvanishing surface terms, we arrive at

$$
\begin{aligned}
\mathscr{I}= & \frac{i \pi^{\omega-2} \Gamma(4-\omega)}{4} \oint d^{3} S^{\mu} \frac{\ell_{\| \mu}}{\left(\ell_{\|}^{2}+\Delta\right)^{4-\omega}} \\
& -\frac{6 i \pi^{\omega-2} \Delta}{\Gamma(\omega-1)} \int d^{4} \ell_{\|} \int_{0}^{\infty} d L \frac{L^{2 \omega-3}}{\left(\ell_{\|}^{2}+L^{2}+\Delta\right)^{4}}
\end{aligned}
$$

where the first integral is over the Euclidean spatial components of a 4 -vector on a three-sphere. The surface integral converges in $1<\omega<2$ while the other in $1<\omega<3$. By taking the surface integral on a three-sphere with radius $R$ and eventually taking the limit $R \rightarrow \infty$ we find

$$
\oint d^{3} S^{\mu} \frac{\ell_{\| \mu}}{\left(\ell_{\|}^{2}+\Delta\right)^{4-\omega}}=2 \pi^{2} \lim _{R \rightarrow \infty} R^{2 \omega-4}
$$

which now converges in the region $\omega \leq 2$ that is, it includes the point $\omega=2$. For $\omega<2$ this surface term vanishes while for $\omega=2$ there is a finite piece, $2 \pi^{2}$, remaining. This is exactly the term that spoils gauge invariance and the equivalence theorem. In DR this term is axiomatically absent-allowing the shifting of integral momenta is among DR's main properties.

Turning into the second integral of (B.7) we note first that the region of convergence includes now $\omega=2$. It gives

$$
\int d^{4} \ell_{\|} \int_{0}^{\infty} d L \frac{L^{2 \omega-3}}{\left(\ell_{\|}^{2}+L^{2}+\Delta\right)^{4}}=\frac{\pi^{2}}{12} \frac{\Gamma(\omega-1) \Gamma(3-\omega)}{\Delta^{3-\omega}} .
$$

By placing (B.8) and (B.9) into (B.7) we finally arrive at (12).

\section{Acknowledgments}

The authors are grateful to Adrian Signer for illuminating discussions and critical comments on the manuscript. The authors would also like to thank R. Pittau, R. Jackiw, F. Piccinini, P. Kanti, S. Martin, and K. Tamvakis for discussions and comments. The authors also thank C. Coriano for bringing to our attention [51]. This research Project is cofinanced by the European Union-European Social Fund (ESF) and National Sources, in the framework of the program "ARISTEIA" of the "Operational Program Education and Lifelong Learning" of the National Strategic Reference Framework (NSRF) 2007-2013. Kristaq Suxho acknowledges full the financial support from the Greek State Scholarships Foundation (I.K.Y).

\section{References}

[1] P. W. Higgs, "Broken symmetries and the masses of gauge bosons," Physical Review Letters, vol. 13, no. 16, pp. 508-509, 1964.

[2] F. Englert and R. Brout, "Broken symmetry and the mass of gauge vector mesons," Physical Review Letters, vol. 13, no. 9, pp. 321-323, 1964.

[3] G. S. Guralnik, C. R. Hagen, and T. W. B. Kibble, "Global conservation laws and massless particles," Physical Review Letters, vol. 13, no. 20, pp. 585-587, 1964.

[4] J. F. Gunion, H. E. Haber, G. L. Kane, and S. Dawson, "The Higgs hunter's guide," Frontiers of Physics, vol. 80, pp. 1-448, 2000.

[5] A. Djouadi, "The anatomy of electroweak symmetry breaking. I: the Higgs boson in the standard model," Physics Reports, vol. 457, no. 1-4, pp. 1-216, 2008.

[6] G. Aad, T. Abajyan, B. Abbott et al., "Observation of a new particle in the search for the standard model Higgs boson with the ATLAS detector at the LHC," Physics Letters B, vol. 716, no. 1, pp. 1-29, 2012.

[7] S. Chatrchyan, V. Khachatryan, A. M. Sirunyan et al., "Observation of a new boson at a mass of $125 \mathrm{GeV}$ with the CMS experiment at the LHC," Physics Letters B, vol. 716, no. 1, pp. 30-61, 2012.

[8] S. Weinberg, "A model of leptons," Physical Review Letters, vol. 19, no. 21, pp. 1264-1266, 1967.

[9] S. L. Glashow, "Partial-symmetries of weak interactions," Nuclear Physics, vol. 22, no. 4, pp. 579-588, 1961. 
[10] A. Salam, "Elementary particle theory," in Proceedings of the 8th Nobel Symposium, N. Svartholm, Ed., p. 367, Wiley, New York, NY, USA, 1968.

[11] J. Ellis, M. K. Gaillard, and D. V. Nanopoulos, "A phenomenological profile of the Higgs boson," Nuclear Physics B, vol. 106, pp. 292-340, 1976.

[12] B. Ioffe and V. A. Khoze, "What can be expected from experiments on colliding $e^{+} e^{-}$beams with energy approximately equal to 100-GeV?" Soviet Journal of Particles and Nuclei, vol. 9, p. 50, 1978.

[13] M. A. Shifman, A. Vainshtein, M. Voloshin, and V. I. Zakharov, "Low-energy theorems for Higgs boson couplings to photons," Soviet Journal of Nuclear Physics, vol. 30, pp. 711-716, 1979.

[14] M. B. Gavela, G. Girardi, C. Malleville, and P. Sorba, "A nonlinear $R_{\zeta}$ gauge condition for the electroweak $S U(2) \times U(1)$ model," Nuclear Physics B, vol. 193, no. 1, pp. 257-268, 1981.

[15] D. Huang, Y. Tang, and Y.-L. Wu, "Note on Higgs Decay into Two Photons $H \rightarrow \gamma \gamma$," Communications in Theoretical Physics, vol. 57, pp. 427-434, 2012.

[16] H. Shao, Y. Zhang, and K. Chao, "Reduction schemes in cutoff regularization and Higgs decay into two photons," Journal of High Energy Physics, vol. 2012, no. 1, article 053, 2012.

[17] F. Bursa, A. Cherman, T. C. Hammant, R. R. Horgan, and M. Wingate, "Calculation of the one W loop $H \rightarrow \gamma \gamma$ decay amplitude with a lattice regulator," Physical Review D, vol. 85, Article ID 093009, 2012.

[18] F. Piccinini, A. Pilloni, and A. Polosa, " $H \rightarrow \gamma \gamma$ : a comment on the Indeterminacy of non-gauge-invariant integrals," Chinese Physics C, vol. 37, no. 4, Article ID 043102.

[19] W. J. Marciano, C. Zhang, and S. Willenbrock, "Higgs decay to two photons," Physical Review D, vol. 85, no. 1, Article ID 013002, 10 pages, 2012.

[20] J. M. Cornwall, D. N. Levin, and G. Tiktopoulos, "Derivation of gauge invariance from high-energy unitarity bounds on the S matrix," Physical Review D, vol. 10, no. 4, pp. 1145-1167, 1974.

[21] C. E. Vayonakis, "Born helicity amplitudes and cross-sections in non-Abelian gauge theories," Lettere Al Nuovo Cimento Series 2, vol. 17, no. 11, pp. 383-387, 1976.

[22] B. W. Lee, C. Quigg, and H. B. Thacker, "Weak interactions at very high energies: the role of the Higgs-boson mass," Physical Review D, vol. 16, no. 5, pp. 1519-1531, 1977.

[23] S. Weinberg, "General theory of broken local symmetries," Physical Review D, vol. 7, no. 4, pp. 1068-1082, 1973.

[24] S. L. Adler, "Axial-vector vertex in spinor electrodynamics," Physical Review, vol. 177, no. 5, pp. 2426-2438, 1969.

[25] J. Bell and R. Jackiw, "A PCAC puzzle: $\pi^{0} \rightarrow \gamma \gamma$ in the sigma model," Il Nuovo Cimento A, vol. 60, pp. 47-61, 1969.

[26] L. Rosenberg, "Electromagnetic interactions of neutrinos," Physical Review, vol. 129, no. 6, pp. 2786-2788, 1963.

[27] A. Dedes and K. Suxho, "Heavy fermion non-decoupling effects in triple gauge boson vertices," Physical Review D, vol. 85, no. 9, Article ID 095024, 31 pages, 2012.

[28] R. Jackiw, "When radiative corrections are finite, but undetermined," International Journal of Modern Physics B, vol. 14, no. 19-20, pp. 2011-2021, 2000.

[29] G. 't Hooft and M. Veltman, "Regularization and renormalization of gauge fields," Nuclear Physics B, vol. 44, no. 1, pp. 189-213, 1972.

[30] J. C. Collins, Renormalization, Cambridge Monographs on Mathematical Physics, Cambridge University Press, Cambridge, UK, 1984.
[31] R. Pittau, "A four-dimensional approach to quantum field theories," Journal of High Energy Physics, vol. 2012, p. 151, 2012.

[32] R. Gastmans, S. L. Wu, and T. T. Wu, "Higgs decay $H \rightarrow \gamma \gamma$ through a W loop: difficulty with dimensional regularization," http://arxiv.org/abs/1108.5322.

[33] R. Gastmans, S. L. Wu, and T. T. Wu, "Higgs decay into two photons, revisited," http://arxiv.org/abs/1108.5872.

[34] J. M. Jauch and F. Rohrlich, The Theory of Photons and Electrons, Springer, New York, NY, USA, 1976.

[35] R. E. Pugh, "Origin shifts in divergent Feynman integrals," Canadian Journal of Physics, vol. 47, pp. 1263-1269, 1969.

[36] V. Elias, G. McKeon, and R. B. Mann, "Shifts of integration variable within four- and n-dimensional Feynman integrals," Physical Review D, vol. 28, no. 8, pp. 1978-1992, 1983.

[37] M. E. Peskin and D. V. Schroeder, An Introduction to Quantum Field Theory, Addison-Wesley, Reading, Mass, USA, 1995.

[38] J. Bagger and C. Schmidt, "Equivalence theorem redux," Physical Review D, vol. 41, no. 1, pp. 264-270, 1990.

[39] M. Shifman, A. Vainshtein, M. B. Voloshin, and V. Zakharov, "Higgs boson decay into two photons through the W-boson loop: No decoupling in the $m_{W} \rightarrow 0$ limit," Physical Review D, vol. 85, no. 1, Article ID 013015, 2012.

[40] F. Jegerlehner, "Comment on $H \rightarrow \gamma \gamma$ and the role of the decoupling theorem and theequivalence theorem," http://arxiv .org/abs/1110.0869.

[41] T. Appelquist and J. Carazzone, "Infrared singularities and massive fields," Physical Review D, vol. 11, no. 10, pp. 2856-2861, 1975.

[42] J. Abdallah, P. Abreu, W. Adam et al., "Measurements of CPconserving trilinear gauge boson couplings WWV $(\mathrm{V} \equiv \gamma, \mathrm{Z})$ in $e^{+} e^{-}$collisions at LEP2," The European Physical Journal C, vol. 66, pp. 35-56, 2010.

[43] J. M. Cornwall, D. N. Levin, and G. Tiktopoulos, "Uniqueness of spontaneously broken gauge theories," Physical Review Letters, vol. 30 , no. 25, pp. 1268-1270, 1973.

[44] M. S. Chanowitz and M. K. Gaillard, "The TeV physics of strongly interacting W's and Z's," Nuclear Physics B, vol. 261, pp. 379-431, 1985.

[45] G. J. Gounaris, R. Kögerler, and H. Neufeld, "Relationship between longitudinally polarized vector bosons and their unphysical scalar partners," Physical Review D, vol. 34, no. 10, pp. 3257-3259, 1986.

[46] S. Dawson and S. Willenbrock, "Radiative corrections to longitudinal-vector-boson scattering," Physical Review D, vol. 40, no. 9, pp. 2880-2887, 1989.

[47] A. Vainshtein, V. I. Zakharov, and M. A. Shifman, "Higgs particles," Soviet Physics Uspekhi, vol. 23, pp. 429-449, 1980.

[48] B. A. Kniehl and M. Spira, "Low-energy theorems in Higgs physics," Zeitschrift für Physik C, vol. 69, no. 1, pp. 77-87, 1995.

[49] A. Pilaftsis, "Higgs-boson low-energy theorem and compatible gauge-fixing conditions," Physics Letters B, vol. 422, no. 1-4, pp. 201-211, 1998.

[50] C. G. Callan Jr., S. Coleman, and R. Jackiw, "A new improved energy-momentum tensor," Annals of Physics, vol. 59, no. 1, pp. $42-73,1970$.

[51] J. Horejsi and M. Stohr, "Higgs decay into two photons, dispersion relations and trace anomaly," Physics Letters B, vol. 379, pp. 159-162, 1996.

[52] S. L. Adler, J. C. Collins, and A. Duncan, "Energy-momentumtensor trace anomaly in spin-1/2 quantum electrodynamics," Physical Review D, vol. 15, no. 6, pp. 1712-1721, 1977. 
[53] M. Klute, R. Lafaye, T. Plehn, M. Rauch, and D. Zerwas, "Measuring higgs couplings from LHC data," Physical Review Letters, vol. 109, no. 10, Article ID 101801, 10 pages.

[54] P. P. Giardino, K. Kannike, M. Raidal, and A. Strumia, "Is the resonance at $125 \mathrm{GeV}$ the Higgs boson?" Physics Letters B, vol. 718, no. 2, pp. 469-474, 2012. 

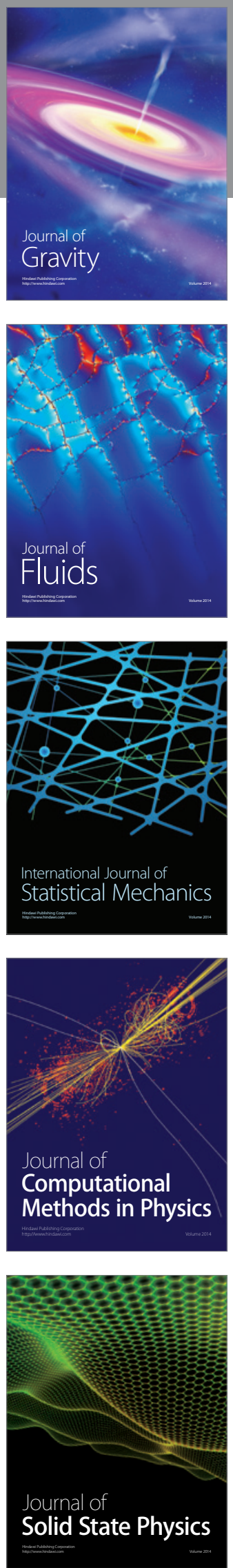

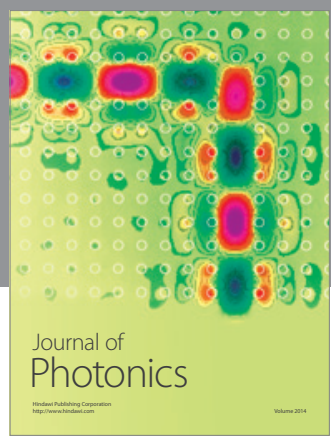

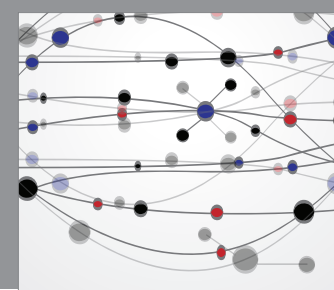

The Scientific World Journal

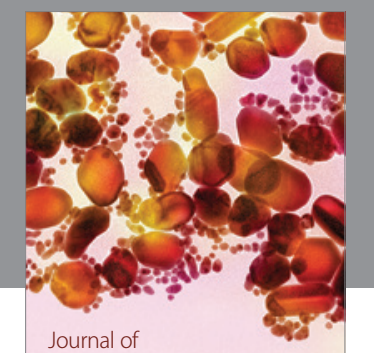

Soft Matter
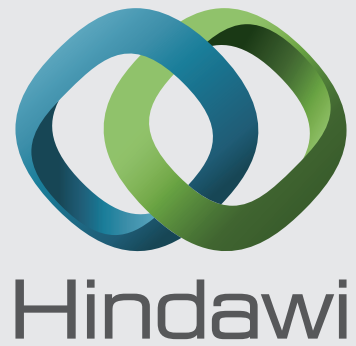

Submit your manuscripts at

http://www.hindawi.com
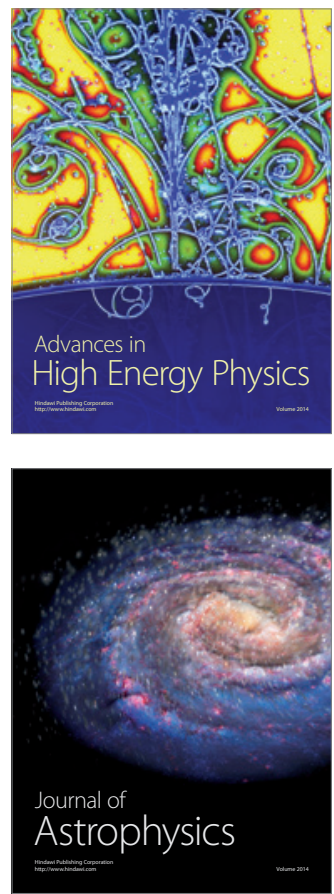
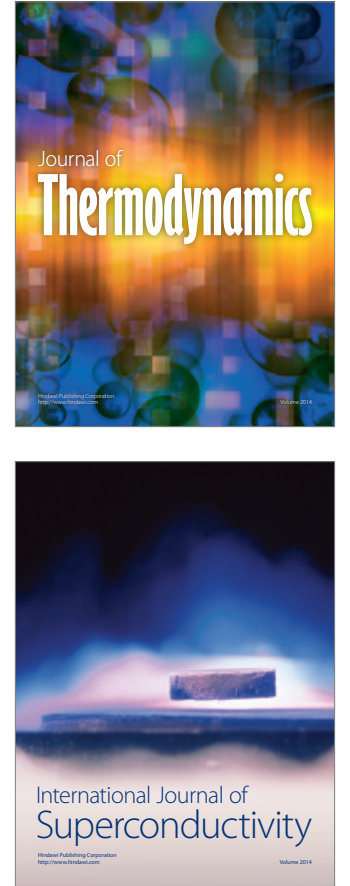
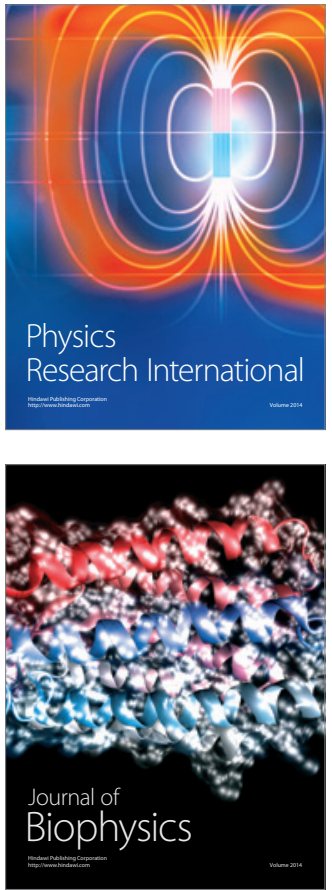
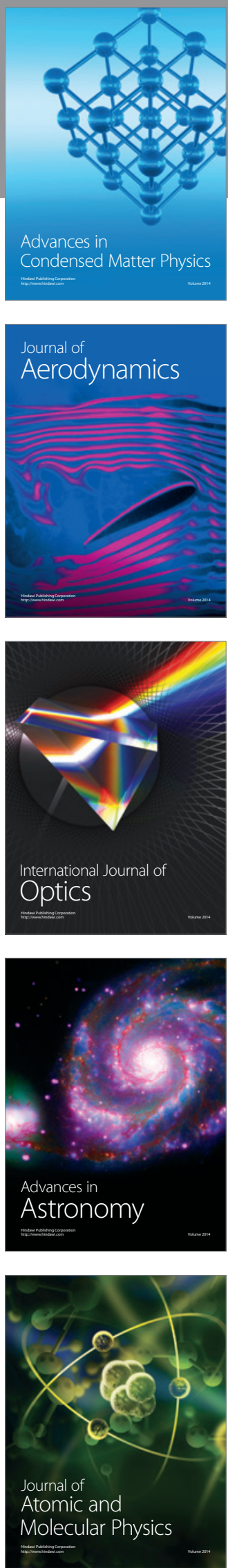